\title{
Consumo de antidepresivos en Chile entre 1992 y 2004
}

\author{
Marcela Jirón',a, Márcio Machado2,b, Inés Ruiz ${ }^{1, c}$. \\ Consumption of antidepressants \\ in Chile from 1992 to 2004
}

Background: Data from the Ministry of Health show that in Chile in $2004,17 \%$ of the population had some form of depression and mood disorders are the tenth cause of disability-adjusted life years (DALY) loss. Aim: To determine consumption of antidepressants (ADs) in Chile from 1992 to 2004. Material and methods: National sales data were obtained from the company IMS Health Chile and converted into defined daily doses (DDDs) per 1,000 inhabitants per day. Available ADs were classified in four pharmacological groups (i.e., serotonin-norepinephrine reuptake inhibitors, SNRIs; selective-serotonin reuptake inhibitors, SSRIs; tricyclic antidepressants, TCAs; and others). Total economic burden of ADs utilization and cost per DDDs were also calculated. Trends over time were analyzed using Pearson-R2. Results: Total ADs consumption in Chile measured by DDDs per 1,000 inhabitants per day (DHD) increased linearly $(y=0.901 x+1.9129$; $R 2=0.9296 ; p<0.001)$ from 2.5 in 1992 to 11.7 in 2004 (total growth of $470.2 \%$ ). SSRIs were the drug class with higher consumption, and fluoxetine the most commonly consumed antidepressant. SSRIs were the drugs that dominated the market representing $79 \%$ of the total drug consumption throughout the years. Total economic burden of ADs in Chile (total cost of DDDs consumed) increased from US\$65.4 million in 2001 to US\$74.6 million in 2004 (14\% increase). Average cost per DDD of all AD increased linearly, however not significantly, from US\$ 0.94 in 2001 to US\$ 1.04 in 2004 $(\mathrm{y}=0.0362 \mathrm{x}+0.8784 ; \mathrm{R} 2=0.7382 ; \mathrm{p}=0,262$ ). Conclusions: DDDs per 1,000 inhabitants per day increased linearly over $470 \%$ from 1992-2004. SSRIs were the most commonly consumed drugs in Chile. Future research should evaluate the cost-effectiveness of antidepressants in Chile, comparing the results with drug utilization, and determining if unnecessary expenditures have been paid out (Rev Méd Chile 2008; 136: 1147-54).

(Key words: Antidepressive agents; Fluoxetine; Mood disorders)

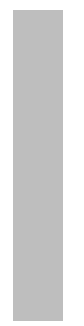
Recibido el 6 de diciembre, 2007. Aceptado el 2 de mayo, 2008.
Conflicto de intereses/financiamiento: Los autores de este artículo no tienen conflicto de intereses. Esta investigación no fue financiada externamente.
${ }^{1}$ Departamento de Ciencia y Tecnología Farmacéutica, Facultad de Ciencias Químicas y Farmacéuticas, Universidad de Chile. Santiago de Chile. ${ }^{2}$ Department of Pharmacy Administration, Leslie Dan Faculty of Pharmacy, University of Toronto, Canadá.
aQuímico Farmacéutico, Magíster en Epidemiología Clínica
bFarmacéutico, Doctor en Ciencias Farmacéuticas
'Químico Farmacéutico

Correspondencia a: Marcela Jirón. Departamento de Cien-

cia y Tecnología Farmacéutica, Facultad de Ciencias

Químicas y Farmacéuticas, Universidad de Chile. Casilla

233, Santiago 1. Fax: 9782990. E mail: mjiron@ciq.uchile.cl 
$\mathrm{E}_{\mathrm{n}}^{\mathrm{n}}$ todos los países la utilización de medicamentos depende de muchos factores, entre los que se encuentran los hábitos de prescripción, la disponibilidad de los productos, las regulaciones sobre medicamentos, las características del sistema de salud y aspectos socioculturales y socioeconómicos de la población. Por lo tanto, es muy difícil extrapolar los datos obtenidos para un país a otro.

Si se desea comparar los datos de utilización de medicamentos, es necesario usar unidades de medición que no sean afectadas por los precios, las formas farmacéuticas por envase, el tamaño de la población, el área geográfica o las instituciones de salud. Para permitir la comparación de estudios nacionales de utilización, la Organización Mundial de la Salud (OMS) promueve el uso de las dosis diarias definidas (DDDs) por 1.000 habitantes por día ${ }^{1}$. Esta organización también establece que los datos de utilización, expresados en DDD por 1.000 habitantes por día (DHD), pueden contribuir para el desarrollo de políticas que promuevan el uso racional de medicamentos, modifiquen patrones de prescripción y hábitos de uso, como también, para evaluar intervenciones educativas, de promoción o regulación ${ }^{1}$. Chile es un país en transición epidemiológica, por lo cual existe una compleja situación de salud en la que algunas enfermedades se asocian a subdesarrollo, mientras otras están relacionadas con estilos de vida de la población y desarrollo económico. Las enfermedades más prevalentes en Chile son las crónicas (hipertensión, diabetes mellitus, otras), cáncer, accidentes automovilísticos y problemas de salud mental $^{2}$. Los trastornos depresivos constituyen una importante prioridad para la salud mental en Chile $^{3}$. En el año 2004, los datos oficiales del Ministerio de Salud de Chile, muestran que 17,5\% de la población presentaba alguna forma de depresión ${ }^{4}$. Adicionalmente, este Ministerio ha informado que los trastornos depresivos ocupan el décimo lugar de las causas principales de discapacidad, expresada en años de vida ajustados por discapacidad (DALYs) ${ }^{2}$. Otros datos muestran que en Santiago los trastornos depresivos son la quinta causa entre las principales asociadas a DALYs y en segundo lugar, cuando este índice se aplica a las mujeres ${ }^{3}$. Un reciente estudio chileno encontró que $31,5 \%$ de la población ha presentado un trastorno psiquiátrico en su vida y $22,2 \%$ ha tenido algún tipo de trastorno mental en los últimos 12 meses $^{5}$.

En atención primaria se ha encontrado que la prevalencia de depresión en Santiago es de 29,5\%. Esta prevalencia es mayor a la encontrada en otras ciudades, como Río de Janeiro (15,8\%) y París $(13,7 \%)^{6}$. Además, para las mujeres que viven en Santiago, el riesgo relativo para desarrollar depresión es 4,73 en relación a los hombres ${ }^{6}$.

En el mercado chileno existen numerosos productos para tratar los trastomos depresivos. Algunos de ellos corresponden a ADs muy conocidos, como la venlafaxina, sertralina, citalopram, amitriptilina, imipramina, entre otros. Además se dispone de productos naturales, como por ejemplo, la hierba de San Juan (Hypericum perforatum). Es sabido que los ADs son ampliamente usados y que los pacientes deben ser monitorizados en forma frecuente para evaluar los efectos logrados. También, tal como ocurre con otros tratamientos, los médicos deben informar a los pacientes sobre los beneficios y riesgos de las diferentes alternativas terapéuticas ${ }^{7}$. Sin embargo en Chile, debido a diferentes razones y aun cuando la mayoría de los medicamentos deben venderse bajo receta médica, aproximadamente $74 \%$ de la población puede obtenerlos sin presentar una receta médica ${ }^{8}$. Debido a la alta prevalencia de trastornos depresivos en la población chilena, pareció interesante analizar la tendencia de consumo de ADs, como también, la carga económica en el período 1992-2004.

\section{MÉtodos}

Los ADs se clasificaron en cuatro grupos: inhibidores selectivos de recaptación de serotonina (ISRSs), inhibidores de recaptación de serotonina y norepinefrina (IRSNs), tricíclicos (TCs) y otros.

Los datos de utilización se estimaron a partir de las ventas nacionales registradas por el IMS Health Database ${ }^{9}$ (de 1992 a 2004) y se convirtieron a número de DDDs consumidas por 1.000 habitantes por día, usando el Indice ATC (Anatomical Therapeutic Chemical) de Clasificación ${ }^{10}$.

El número total de DDDs consumidas para cada medicamento se obtuvo determinando el número total de miligramos consumidos de un medicamento en cada año (número de comprimidos/cápsulas 
de un producto específico $\mathrm{x}$ la cantidad de miligramos en cada comprimido/cápsula $\mathrm{x}$ las ventas totales del producto en cada año) divididos por la DDD específica para cada medicamento. Luego, el número total de DDDs consumidas de cada medicamento se convirtió a 1.000 habitantes por día. Cuando la DDD de un medicamento no estaba disponible se usó la dosis más empleada y la población de cada año se obtuvo del Instituto Nacional de Estadísticas (INE) ${ }^{11}$.

Las ventas de $\mathrm{ADs}$ se convirtieron a costo por cada DDD dividiendo el número total de DDDs consumidas por los costos totales de venta. Adicionalmente, la carga económica de la utilización de ADs se estimó multiplicando el costo promedio por DDD por el número total de DDDs consumidas cada año. Los datos para estimar los costos que estuvieron disponibles fueron los de 2001 a 2004. Los costos se expresaron en dólares ameri- canos (US\$). Los datos se analizaron usando el Statistical Package for Social Sciencies (SPSS), versión 10.0. Los datos de interés, como DDDs/ 1.000 habitantes/día (DHD) y ventas en el mercado, se graficaron en el tiempo y se establecieron tendencias mediante regresión lineal (Pearson- $R^{2}$ ).

\section{RESULTADOS}

Cuando se realizó el estudio había 12 antidepresivos disponibles en el mercado chileno. A través del período de estudio se introdujeron varios agentes, como por ejemplo, la paroxetina en 1993, el citalopram en 1995, la mirtazapina en 1997 y la venlafaxina en 1999. El número total de ADs disponibles en Chile en 2004 era 20. La Tabla 1 muestra los ADs disponibles en el mercado chileno durante el período en estudio.

Tabla 1. D isponibilidad de antidepresivos en el mercado chileno durante 1992-2004

\begin{tabular}{|llc|}
\hline Clase fármaco & Fármaco & Disponibilidad (años) \\
\hline ISRSs & Citalopram & $1995-2004$ \\
& Escitalopram & $2003-2004$ \\
& Fluoxetina & $1992-2004$ \\
& Paroxetina & $1993-2004$ \\
SRSNs & Fertralina & $1992-2004$ \\
& Fluvoxamina & $2001-2004$ \\
Tricíclicos & Venlafaxina & $1999-2004$ \\
& Milnacipran & $2004-2004$ \\
& Amineptina & $1992-2002$ \\
& Amitriptilina & $1992-2004$ \\
& Clomipramina & $1992-2004$ \\
& Desipramina & $2001-2004$ \\
Otros & Imipramina & $1992-2004$ \\
& Maprotilina & $1992-2004$ \\
& Trimipramina & $1992-2004$ \\
& Bupropion & $2000-2004$ \\
& Mianserina & $1992-2004$ \\
& Mirtazapina & $1997-2004$ \\
& Moclobemida & $1992-2004$ \\
& Opripramol & $1992-1996$ \\
& Tianeptina & $2001-2003$ \\
& Trazodona & $1992-2004$ \\
& Reboxetina & $2001-2004$ \\
\hline
\end{tabular}

ISRSs, Inhibidores Selectivos de la Recaptación de Serotonina; IRSN, Inhibidores de la Recaptación de Serotonina y Noradrenalina. 
La Figura 1 muestra las DHDs según grupo de ADs. El consumo total de ADs en Chile, en el período 1992-2004, expresado en DHD aumentó en forma lineal $\left(y=0,901 x+1,9129\right.$; $R^{2}=0,9296$; $p$ $<0,001)$. El consumo total de DDDs por 1.000 habitantes por día aumentó desde 2,5 en 1992 a 11,7 en 2004, representando un crecimiento de $470,2 \%$.

Durante todo el período de estudio los ADs más consumidos fueron los ISRSs, representando $59,5 \%$ del total de DHDs, seguidos por los TCs (33,7\%) y el grupo de "otros" (6,9\%). En 2004, los ISRSs fueron nuevamente el grupo más consumido (78,9\%), seguido por el de TCs $(13,7 \%)$ y el de "otros" (4,3\%). Los IRSNs explicaron 3,1\% del consumo total de ADs en 2004. Según medicamentos en particular, la fluoxetina fue la más consumida, representando $59 \%$ y $48 \%$ del total de DHDs en 1992 y 2004, respectivamente.

En la Tabla 2 se presenta la expansión/ reducción y la participación en el consumo por total de DDDs. Los ISRSs dominaron el mercado con un promedio de $79 \%$ del consumo total a través de los años del estudio. La fluoxetina fue el medicamento que más contribuyó (57\%) a esta dominación del total del consumo de ADs. Después del año 2000, se encontró que la utilización de los ISRSs se redujo en 3\%. El AD perteneciente al grupo de los ISRSs que tuvo la mayor expansión en su consumo fue el citalopram ( $64 \%$ en promedio, período 1998 a 2004).
En 2004, los IRSNs representaron 3\% del total de consumo de ADs, mostrando un aumento de utilización de $41 \%$ desde 2000 a 2004, principalmente atribuido al consumo de venlafaxina. El consumo de TCs mostró un leve aumento en 2000 y después de este año, casi no mostraron cambios en el número total de DDDs consumidas. En 2004 el consumo de TCs representó $14 \%$ del total de DDDs de ADs y la utilización de todos estos medicamentos mostró una reducción leve. Los ADs agrupados en "otros" tuvieron un pequeño aumento de su consumo hasta 2002; sin embargo su participación en el consumo en el período 19982004 prácticamente no cambió (4\% en promedio).

La carga económica total del consumo de ADs en Chile (medida mediante el costo del total de DDDs consumidas para cada año) aumentó de US\$ 65,4 millones en 2001 a US\$ 74,6 millones en 2004 (14\% de aumento). El costo promedio por DDD de todos los antidepresivos aumentó de manera lineal, pero no significativa, desde US\$ 0,94 en 2001 hasta US\$ 1,04 millones en 2004 (y $=0,0362 x+0,8784 ; R^{2}=0,7382 ; p=0,262$ ). La Figura 2 muestra el costo promedio por DDD de los ADs en Chile desde el año 2001 hasta 2004.

\section{DISCUSIÓN}

El presente estudio representa el primer análisis chileno relativo a la utilización de varios ADs en el

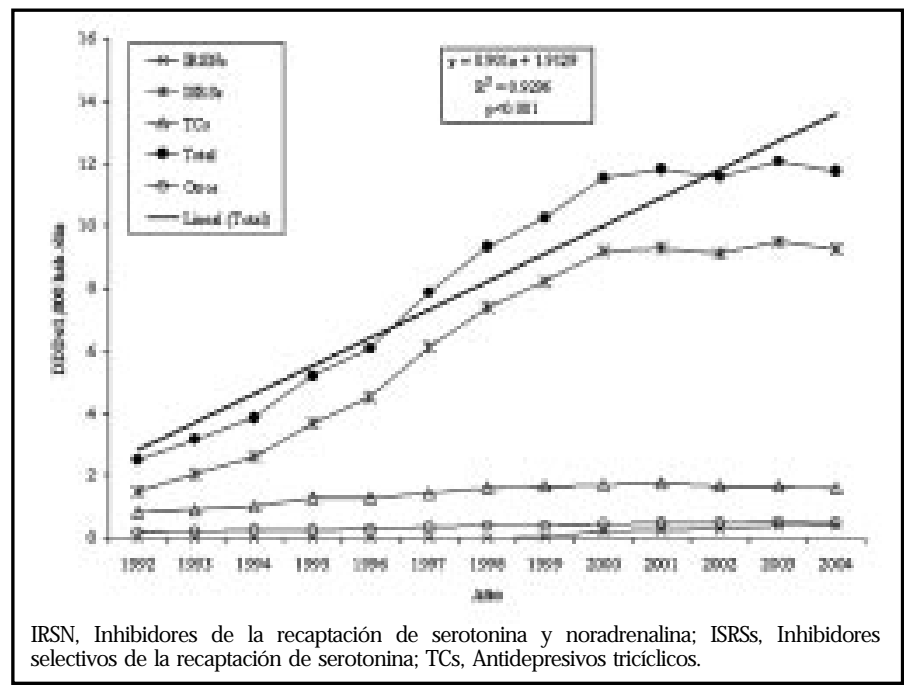

Figura 1. Número total de dosis diarias definidas (DDDs) por 1.000 habitantes por día según clase de fármaco. 


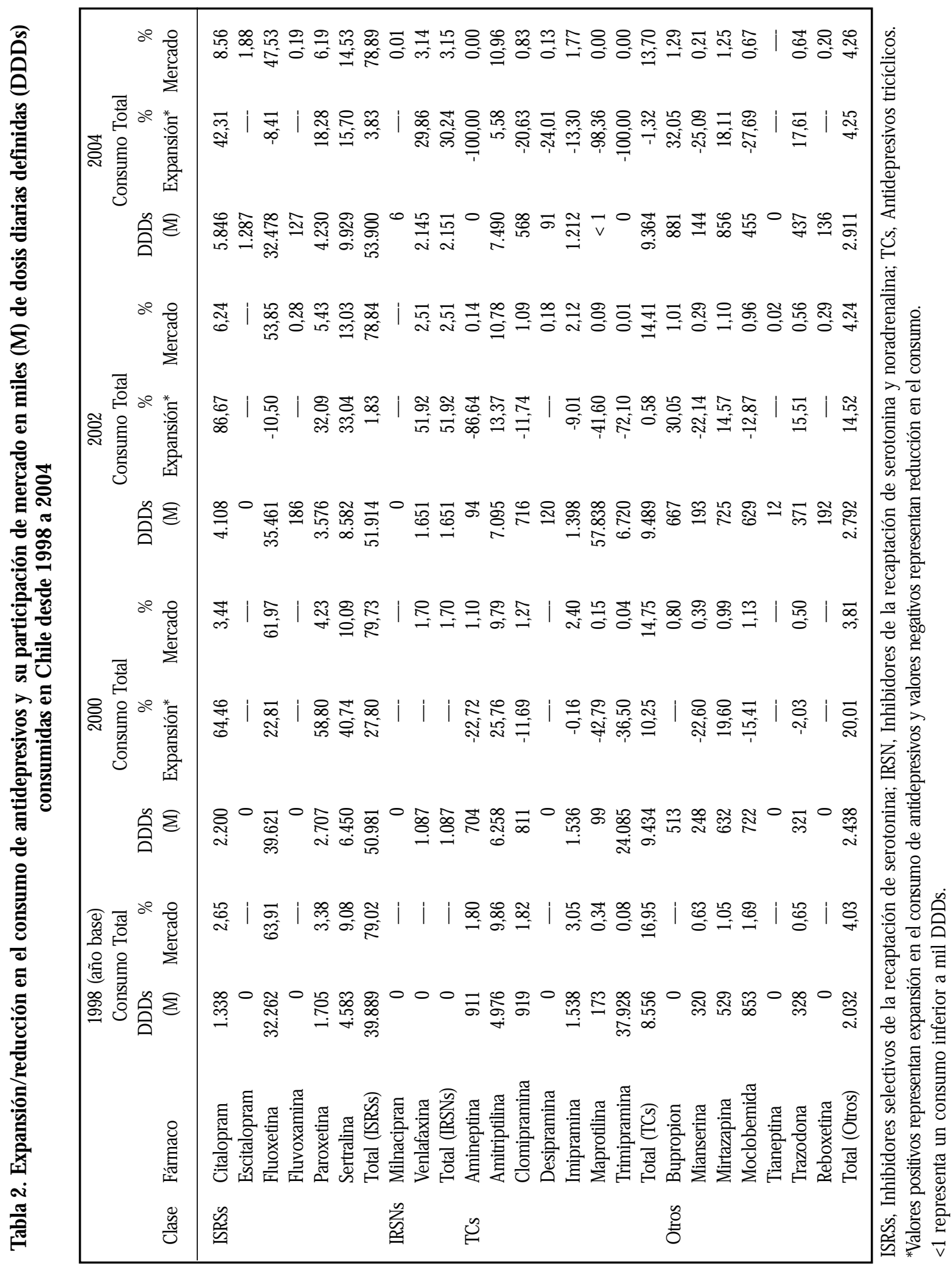




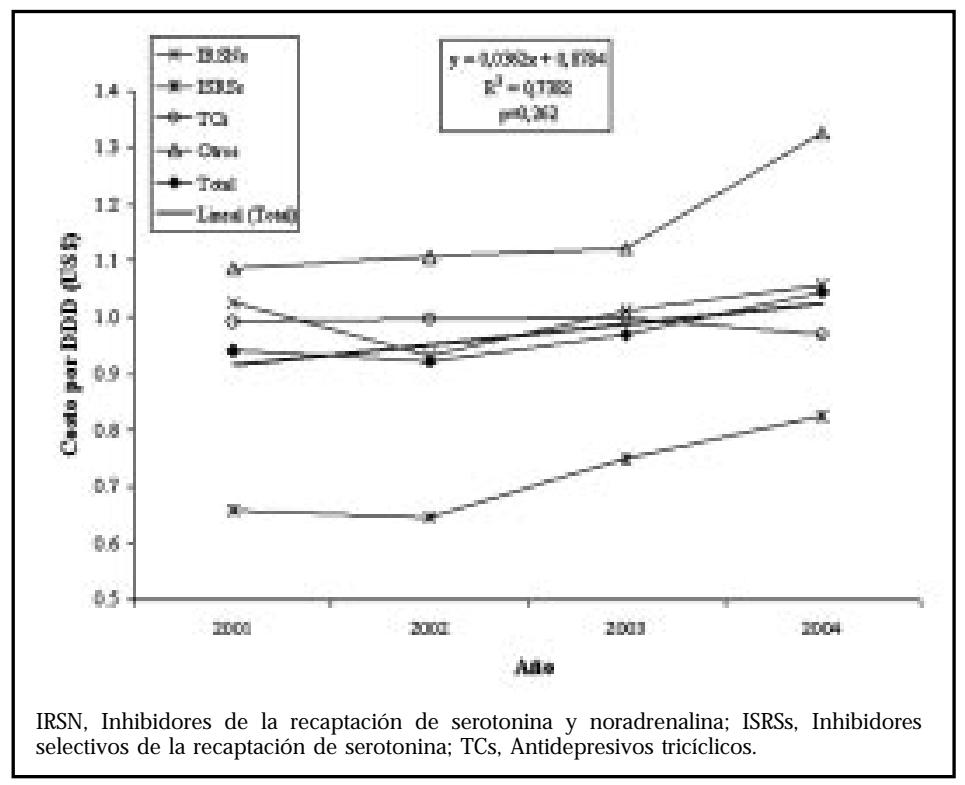

Figura 2. Costo promedio por dosis diaria definida (DDD) de antidepresivos en Chile según clase de fármaco.

período 1992-2004, usando la metodología sugerida por la OMS1. Se evaluó el consumo de ADs antiguos y recientemente introducidos (representados principalmente por los ISRSs y los IRSNs).

Se observó un aumento lineal estadísticamente significativo en la utilización de medicamentos ADs a través del período estudiado. El número de DHDs aumentó más de 470\% entre 1992 y 2004. El aumento en el consumo de ADs está bien documentado en la literatura ${ }^{12}$ y podría deberse a múltiples causas, incluyendo mayor duración de la depresión y de episodios de depresión ${ }^{13}$, aumento en la incidencia/prevalencia de depresión en etapas tempranas de la vida (niños y

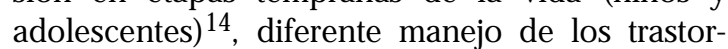
nos depresivos ${ }^{15}$ y cambio de la psicoterapia por farmacoterapia ${ }^{12}$. Adicionalmente, se han descrito nuevas indicaciones para los ADs, especialmente en el caso del bupropion, que también se usa para dejar de fumar. Hemels et $\mathrm{al}^{12}$ comunicaron que el aumento en el consumo de los ISRSs y IRSNs podría estar relacionado con el manejo de otros trastornos mentales, tales como trastornos obsesivos compulsivos, bulimia, trastornos de ansiedad social y trastorno de pánico. Además, los ADs se han usado para tratar diferentes tipos de dolor crónico, tales como dolor músculo-esquelético y neuropático y algunas formas de migraña.

Encontramos que el grupo de los ISRSs fue el que tuvo más influencia en el aumento en el consumo de ADs en Chile. Según medicamento individual, el antidepresivo más consumido fue la fluoxetina. Se han observado consumos similares en Canadá ${ }^{12}$, Francia ${ }^{16}$, Estados Unidos de Norteamérica ${ }^{17}$ y Australia ${ }^{18}$. Recientemente un estudio transversal del consumo de ADs en la población general de Santiago, reportó tendencias similares de uso, como también, serios problemas de adherencia/cumplimiento ${ }^{19}$.

Aunque a través de los años los ISRSs dominaron el mercado chileno de consumo de ADs, puesto que constantemente tuvieron la mayor participación en el consumo, algunos agentes en particular, tales como citalopram y venlafaxina, mostraron un creciente o constante incremento en su uso. Esta situación podría atribuirse a las características farmacológicas de estos medicamentos, que se ha demostrado poseen mejores perfiles de eficacia y seguridad ${ }^{20}$. El escitalopram fue introducido al mercado chileno en 2003 y en ese año su utilización alcanzó a 0,5\% del total del consumo de ADs. 
De modo similar a lo encontrado en el trabajo de Hemels et $\mathrm{al}^{12}$ empleando la misma metodología, en nuestro estudio se encontró que el consumo de los TCs no tuvo variaciones significativas a través de los años. Aun cuando en el mercado se introdujeron nuevos agentes con beneficios terapéuticos importantes, especialmente en lo referente a seguridad, la utilización de ellos se mantuvo con una participación promedio de $20 \%$ del mercado de consumo de ADs durante el período. Este hecho podría deberse a que en la práctica clínica los TCs son los medicamentos de elección cuando ha fallado el tratamiento de titulación con ISRSs o IRSNs para controlar los trastornos depresivos.

En el año 1995 se introdujo una medida regulatoria sobre las benzodiazepinas que estipulaba que para dispensarlas debía existir una prescripción médica ${ }^{21}$. Como resultado, probablemente algunos pacientes que usaban benzodiazepinas pudieron haber recibido prescripción de ADs u otros tal vez comenzaron a usar ADs sin evaluación médica. La carga económica total de ADs en Chile aumentó $14 \%$ en un período de 4 años (2001 a 2004). El costo promedio por DDD para todos los ADs aumentó linealmente desde US\$ 0,94 en 2001 hasta US\$ 1,04 en 2004 ( $p$ $=0,262$ ). Este resultado indica un aumento no significativo de $11 \%$ en los años estudiados. Sin embargo, ese aumento fue similar al del índice chileno general del precio al consumidor (variación acumulativa de 12 meses) para el mismo período y que se estimó en cerca de $10 \% 22$.

De acuerdo a Machado et $\mathrm{al}^{23}$, en Chile no se han realizado estudios farmacoeconómicos de los ADs. En el presente estudio no fue posible hacer adecuadamente un análisis del uso de terapias antidepresivas costo-efectivas para el manejo de las enfermedades mentales en Chile. En un análisis farmacoeconómico realizado en Brasil ${ }^{24}$ los ISRSs no mostraron ser costo-efectivos para el

\section{REFERENCIAS}

1. Bergman U, Grimsson A, Wahba AW, Westerholm B. Studies in Drugs Utilization: Methods and Applications. Regional Publication, European Series № 8, Copenhagen. World Health Organization. Regional Office for Europe. 1979. manejo de los trastornos depresivos mayores al encontrarse el mayor costo por paciente exitosamente tratado (costo por remisión completa de síntomas). $\mathrm{Si}$ se asume que estos datos son transferibles a Chile, el alto uso observado de los ISRSs a través de los años en el país podría generar gastos innecesarios tanto para el sistema público como para el privado.

El presente estudio tiene algunas limitaciones. La más importante se relaciona con los datos obtenidos para realizar el análisis. Los datos del IMS Chile representan una extrapolación de los obtenidos en una muestra de farmacias comunitarias y no incluyen datos de utilización hospitalaria. Por lo cual, el presente análisis es conservador, principalmente al calcular la carga económica de los ADs en Chile. También y debido a la falta de datos de años anteriores, el aumento lineal observado para el costo promedio por DDD podría no alcanzar significación estadística. Adicionalmente, las DDD entregadas por el ATC podrían no ser representativas de las dosis efectivamente empleadas en poblaciones especiales, como pacientes pediátricos o geriátricos. Aun más, los gastos en medicamentos no siempre representan el uso de ellos. En conclusión, en el presente estudio se encontró que las DDDs por 1.000 habitantes por día de ADs aumentó linealmente por sobre 470\% en Chile en el período 1992-2004. El grupo más utilizado fue el de los ISRSs y el AD más consumido, la fluoxetina. A través de los años, los antidepresivos más recientes mostraron un aumento o expansión constante en su consumo. La carga económica total de los ADs en Chile aumentó $14 \%$ en los últimos cuatro años del período analizado en el estudio. Se necesita realizar investigaciones que evalúen la relación costo-efectividad de las terapias antidepresivas en Chile y que se comparen con los estudios de utilización con el fin de establecer si se han realizado gastos innecesarios.

2. Situación de Salud en Chile 1999. Tarjeta de presentación. Ministerio de Salud. Chile. 2000.

3. Ministerio de Salud. División de Salud de las Personas. Programa de Salud del Adulto. MINSAL, Santiago, Chile. 1999.

4. Encuesta Nacional de Salud 2004. Ministerio de Salud. Chile. 2004. 
5. Vicente B, Kohn R, Rioseco P, Saldivia S, Torres S. Lifetime and 12-Month Prevalence of DSM-III-R Disorders in the Chile Psychiatric Prevalence Study. Am J Psychiatry 2006; 163: 1362-70.

6. Florenzano R, Acuña J, Fuluerton C, Castro C. Estudio comparativo de frecuencia y características de los trastornos emocionales en pacientes que consultan en el nivel primario de atención en Santiago de Chile. Rev Méd Chile 1998; 126: 397-405.

7. COMpton MT, Nemeroff CB. The Evaluation and Treatment of Depression in Primary Care. Clin Cornestone 2001; 3: 10-22.

8. Situación del medicamento en Chile, División de Salud de las Personas, Departamento Programa de las Personas. Ministerio de Salud. Chile. 1999.

9. IMS Health Database. www.imshealth.com

10. ATC classification index with DDDs: Guidelines for ATC classification and DDD assignment. WHO Collaborating Centre for Drug Statistics Methodology. January 2004.

11. Chile: Anuario de Estadísticas Vitales 2000. Instituto Nacional de Estadísticas. Ministerio de Salud. Chile. 2002.

12. Hemels ME, Koren G, Einarson TR. Increased use of antidepressants in Canada: 1981-2000. Ann Pharmacother 2002; 36: 1375-9.

13. Patten SB. The duration of major depressive episodes in the Canadian general population. Chronic Dis Can 2001; 22: 1-6.

14. Stephens T, Joubert N. The economic burden of mental health problems in Canada. Chronic Dis Can 2001; 22: 18-23.

15. Judd LL, Paulus MJ, Schettler PJ et al. Does incomplete recovery from first lifetime major depressive episode herald a chronic course of illness? Am J Psychiatry 2000; 157: 1501-4.
16. Ole JP, Elomari F, Spadone C, Lepine JP. Antidepressants consumption in the global population in France. Encephale 2002; 28: 411-7.

17. MA J, LeE KV, StaFFoRd RS. Depression treatment during outpatient visits by U.S. children and adolescents. J Adolesc Health 2005; 37: 434-42.

18. McManus P, Mant A, Mitchell PB, Montgomery WS, Marley J, Auland ME. Recent trends in the use of antidepressant drugs in Australia, 1990-1998. Med J Aust 2000; 173: 458-61.

19. Jirón M. Prevalencia de consumo de antidepresivos en la población mayor de 15 años de Santiago. Tesis (Magíster en Ciencias, mención Epidemiología Clínica). Santiago, Chile. Universidad de Chile. Facultad de Medicina, 2004. $88 \mathrm{~h}$.

20. Machado M, Iskedjian M, Ruiz I, Einarson TR. Remission, dropouts, and adverse drug reaction rates in major depressive disorder: a meta-analysis of headto-head trials. Curr Med Res Opin 2006; 22: 1825-37.

21. Decreto Supremo 1506. Ministerio de Salud de Chile. Modifica reglamento de estupefacientes y psicotrópicos. Diario Oficial 30 de septiembre de 1993.

22. Banco Central de Chile. Base de Datos Estadísticos. Available at: http://si2.bcentral.cl/Basededatoseconomicos (consultado el 3 de septiembre de 2006)

23. Machado M, ISKEDJian M, EinarSON TR. Quality assessment of published health economic analyses from South America. Ann Pharmacother 2006; 40: 943-9.

24. Machado M, Iskedjian M, Ruiz I, Einarson TR. The Economic Impact of Introducing Serotonin-Noradrenaline Reuptake Inhibitors into the Brazilian National Drug Formulary: Cost-Effectiveness and Budget-Impact Analyses. Pharmacoeconomics 2007; 25: 979-90. 